\title{
New Analysis Framework for Transient Stability Evaluation of DC Microgrids
}

\author{
Yanghong Xia, Member, IEEE, Wei Wei, Teng Long, Member, IEEE, Frede Blaabjerg, Fellow, IEEE \\ and Peng Wang, Fellow, IEEE
}

\begin{abstract}
Because of the low inertia of dc microgrids, system state variables are easily changed acutely after being disturbed. Hence, dc microgrids meet the serious transient stability issues especially for some stressed states. But the transient stability analysis is a very challenging problem since the dc microgrid system is high-order and nonlinear. To offer a new and more effective analysis framework, this paper proposes a nonlinear decoupling method to evaluate the transient stability of dc microgrids. The proposed nonlinear decoupling method takes full consideration of the nonlinearity of the dc microgrid system and approximately transforms the original nonlinear system into a series of decoupled first-order quadratic or second-order quadratic systems. For these decoupled low-order quadratic systems, their dynamics and stability can be analyzed easily, then the transient stability of the original system can be reflected indirectly. Also, the nonlinear decoupling based analysis framework can be extended to other power electronics dominated power systems to evaluate their transient stability. The accuracy of the proposed analysis method has been validated through related case studies.
\end{abstract}

Index Terms-Transient stability, nonlinear decoupling, DC microgrid, quadratic system, power electronics dominated power system.

\section{INTRODUCTION}

$\mathrm{D}^{\mathrm{c}}$ C microgrids are thought to be an effective solution to integrate distributed renewable energy sources because of their high flexibility and high efficiency [1]-[2]. Being different from the conventional synchronous generators dominated ac power system, the dc microgrid is mainly comprised of power electronic devices to realize energy conversion, which makes the system inertia is relatively low. Then, the system state variables like voltages, currents and so on can be easily changed acutely when the dc microgrid is disturbed. Therefore, compared to small-signal stability issues [3]-[5], the dc microgrid meets more serious transient stability issues. For some stressed states, the transient stability challenges are more

This work is supported in part by the National Key R\&D Program of China (2017YFB0903300), in part by the Project founded by China Postdoctoral Science Foundation (2019M660139)

Y. Xia and T. Long are with the Department of Engineering, University of Cambridge, Cambridge CB2 1TN, U.K. (e-mail: royxiayh@126.com; t1322@cam.ac.uk)

W. Wei is with the College of Electrical Engineering, Zhejiang University, Hangzhou, 310027, China. (e-mail: wwei@zju.edu.cn).

F. Blaabjerg is with the Department of Energy Technology, Aalborg University, 9220 Aalborg, Denmark (e-mail: fbl@et.aau.dk).

P. Wang is with the School of Electrical and Electronic Engineering, Nanyang Technological University, Singapore 639798. (e-mail: epwang @ntu.edu.sg). prominent, which greatly influences the system operation. Hence, it is very urgent to develop effective transient stability analysis methods and reveal the unstable mechanism.

For the transient stability analysis of dc microgrids, the existing methods are mainly based on the Lyapunov's theory [6]-[15]. Lyapunov's theory is to find a proper Lyapunov function. In [6], the T-S multimodeling method is used to generate a Lyapunov function. Based on this function, the region of attraction (ROA) is evaluated. But the computed T-S multimodels will encounter the curse of dimensionality as the nonlinear terms and system orders increase. The mixed potential function is another usually adopted candidate form of Lyapunov function. According to this thought, the corresponding mixed potential models are established in [7]-[8] to analyze the transient stability of the dc microgrid where the dynamics of renewable energy sources are simplified into negative constant power loads (CPLs). Hence, the accuracy of the results is degraded. To decrease the conservatism of the mixed potential function, the characteristics of load or renewable energy source converters are taken into consideration in [9], but the number of nodes that can be dealt with is decreased obviously. In addition, some heuristic methods are also used to determine a proper Lyapunov function. In [10] and [11], the genetic algorithm is used to find an optimal Lyapunov function according to the goal that the estimated ROA is big enough. The high ratio of CPLs will cause the system instability. To decrease the orders, the whole dc microgrid is viewed as an ideal dc voltage source connecting several ideal CPLs in [12], where the dynamics of $\mathrm{dc} / \mathrm{dc}$ converters and their controllers are completely ignored. Based on this simplified model, the semidefinite programming technology is used to evaluate the ROA of the system. Liu et al. [13] pay attention to the robust stability of dc microgrids considering the uncertainty of CPLs. In [14] and [15], the transient (or large-signal) stability analysis tools in microgrids are summarized.

In summary, the use of Lyapunov's theory to analyze the transient stability of dc microgrids is hindered by the system orders. If the nodes in dc microgrids become large or the detailed models of dc microgrids are taken into consideration, the obtained stability results through the Lyapunov's theory is very conservative. At the same time, for the high-order system, the Lyapunov based stability analysis methods cannot give much insight about how the interaction of the system variables affects the stability.

In order to handle the complexity of the high-order nonlinear power system, the decoupling thought based on the norm form and modal series theories is developed [16]-[21], which is very 
effective for the analysis of low-frequency electromechanical oscillations. The procedures of the typical $2^{\text {nd }}-$ order norm form analysis can be summarized as follows [16]-[17]. 1) The model of a power system is approximately presented with $2^{\text {nd }}-$ order nonlinearities. 2) Applying the classical modal transformation, the original system is transformed into modal space ( $\mathrm{Y}$-space), the model in the Y-space still has $2^{\text {nd }}$-order nonlinearities. 3) Through a proper nonlinear transformation, the Y-space is mapped into the $\mathrm{Z}$-space, where the $2^{\text {nd }}$-order nonlinearities are eliminated while the $3^{\text {rd }}$ - or higher order nonlinearities may be introduced because of the nonlinear transformation. Omitting the higher-order nonlinearities, the model in the Z-space is linear and it can reflect the modal interactions. Then, more accurate transient dynamics can be obtained. Also, the accuracy can be further improved with inclusion of higher-order terms [18]-[19]. In addition, the modal interactions can be also studied through the modal series method according to the structure of solutions of differential equations [20]-[21].

No matter norm form analysis or modal series method, the final models in the decoupled state spaces (like Z-space) is still linear, hence more nonlinear dynamics cannot be analyzed like limit cycle, ROA and so on. Therefore, the conventional norm form analysis or modal series method is usually used in analyzing the low-frequency electromechanical oscillations, but cannot explain transient unstable phenomena.

From the above narration, it can be seen that the existing analysis frameworks for transient stability evaluation of dc microgrids still need to be further improved. The obtained stability results based on the existing analysis frameworks cannot provide effective guide for the reliable operation of $\mathrm{dc}$ microgrids, especially when the system is large and the detailed nonlinear dynamics need to be considered. Focusing on this problem, this paper proposes a new analysis framework for transient stability evaluation of dc microgrids based on the nonlinear decoupling to overcome the high-order and nonlinear challenges. In the new framework, the conventional norm form analysis is developed. In the decoupled space, the quadratic nonlinear terms are retained, then the original nonlinear system is approximately transformed into a series of decoupled first-order quadratic or second-order quadratic systems. These decoupled low-order quadratic systems are simple enough but retain some nonlinear nature. Hence, the detailed nonlinear dynamics can be analyzed like evaluating ROA and so on. In this way, the transient stability of the original system can be reflected indirectly and some transient unstable phenomena can be explained. Also, the nonlinear decoupling based analysis framework can be extended to other power electronics dominated power systems to evaluate their transient stability. All the conclusions are verified by the related case studies.

The remainder of this paper is organized as follows. In Section II, the model of the typical dc microgrid is established. In Section III, the fundamental principle of the nonlinear decoupling is introduced. Then, case studies are conducted and discussions are made in Section IV. At last, the conclusions are drawn in Section V.

\section{Modeling OF THE DC Microgrid}

The dc microgrid integrates many dc distributed generations (DGs) and dc loads. The dc DGs include renewable energy sources like PVs and storages, while the dc loads include electric vehicles (EVs), LEDs and so on. These sources and many loads are connected to the dc bus through power electronic devices. Hence, the inertia of the whole dc microgrid is relatively low. Due to the uncertainty of DGs (like PVs) and loads (like EVs), the dc microgrid often suffers some disturbances, which will cause system state variables can be easily changed acutely because of the low inertia, especially in the islanded mode. Hence, the islanded dc microgrid dominated by the PV generators is studied in this paper, whose typical topology is shown in Fig.1.

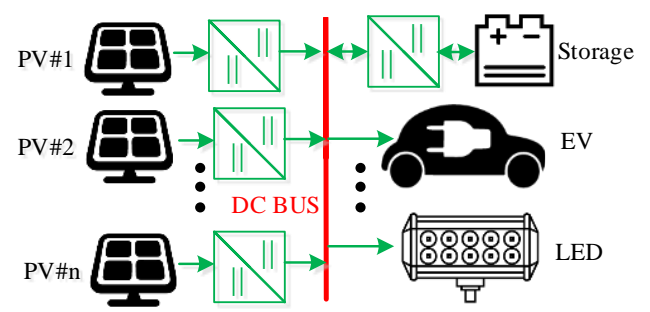

Fig.1. Typical topology of the islanded de microgird.

\section{A. Model of the PV Generator}

The structure of the PV generator and its control is shown in Fig.2. The PV panel is connected to the dc bus through a buck $\mathrm{dc} / \mathrm{dc}$ converter. The filter capacitance on the PV side is $C_{p v}$, the output side is filtered through a LC filter, whose inductance and capacitance are $L_{c}$ and $C_{c}$. The line resistance between the PV generator and the dc bus is $r$. The output voltage and current of the PV panel are $v_{p v}$ and $i_{p v}$. The inductive current and the capacitive voltage on the output side are $i_{c}$ and $v_{o}$.

Usually, the PV generators and other similar renewable energy sources operate in the maximum power point tracking (MPPT) mode to provide power support. Hence, the outer loop is perturbation and observation $(\mathrm{P} \& \mathrm{O})$ based power controller, which can realize MPPT and generate reference value $v_{p v}^{r e f}$ for the inner loop. The inner loop is the proportional-integral (PI) based voltage controller, which makes $v_{p v}$ follow its reference value $v_{p v}^{r e f}$ accurately. Since the bandwidth of power loop (the typical regulation period is $0.1 \mathrm{~s}-1 \mathrm{~s}$ ) is much slower than the voltage loop, the dynamics of power loop is neglected.

Based on Fig.2, the model of the PV generator can be established as

$$
\begin{aligned}
& \frac{d S_{c, m}}{d t}=k_{I c}\left(v_{p v, m}^{r e f}-v_{p v, m}\right) \\
& C_{p v, m} \frac{d v_{p v, m}}{d t}=i_{p v, m}-\left[1-S_{c, m}-k_{P c, m}\left(v_{p v, m}^{r e f}-v_{p v, m}\right)\right] i_{c, m} \\
& L_{c, m} \frac{d i_{c, m}}{d t}=\left[1-S_{c, m}-k_{P c, m}\left(v_{p v, m}^{r e f}-v_{p v, m}\right)\right] v_{p v, m}-v_{o, m} \\
& \left(C_{c, m} \frac{d v_{o, m}}{d t}=i_{c, m}-\frac{v_{o, m}-\sum_{i=1}^{n+1} \alpha_{i} \cdot v_{o, i}}{r_{m}}\right.
\end{aligned}
$$

where $m=1,2, \ldots, n$ presents the $m$-th $\mathrm{PV}$ generator, $S_{c, m}$ is the output of the integral controller, $k_{P c, m}$ and $k_{I c, m}$ are the proportional coefficient and integral coefficient of the PI 
controller, $v_{o, i}$ are the output voltage of the $i$-th node (including the storage node indexed by $n+1), \alpha_{i}=\frac{1 / r_{i}}{1 / R_{L}+\sum_{i=1}^{n+1} 1 / r_{i}}, r_{i}$ is the line resistance between the $i$-th node and the dc bus, $R_{L}$ is the lumped load.

Assuming the equilibrium point is $\left(S_{c, m}^{*}, V_{p v, m}^{*}, I_{c, m}^{*}, V_{o, m}^{*}\right)$, then (1) can be rewritten into

$$
\begin{aligned}
& \begin{aligned}
& \frac{d \Delta S_{c, m}}{d t}=- k_{I c, m} \Delta v_{p v, m} \\
& C_{p v, m} \frac{d \Delta v_{p v, m}}{d t}=\left(g_{p v, m}-k_{P c, m} I_{c, m}^{*}\right) \Delta v_{p v, m}-\left(1-S_{c, m}^{*}\right) \Delta i_{c, m} \\
&+ I_{c, m}^{*} \Delta S_{c, m}-k_{P c, m} \Delta v_{p v, m} \Delta i_{c, m}+\Delta S_{c, m} \Delta i_{c, m}
\end{aligned} \\
& L_{c, m} \frac{d \Delta i_{c, m}}{d t}= \\
& \quad\left[\left(1-S_{c, m}^{*}\right)+k_{P c, m} V_{p v, m}^{*}\right] \Delta v_{p v, m}-\Delta v_{o, m}- \\
& V_{p v, m}^{*} \Delta S_{c, m}-\Delta S_{c, m} \Delta v_{p v, m}+k_{P c, m} \Delta v_{p v, m}^{2} \\
& \left(C_{c, m} \frac{d \Delta v_{o, m}}{d t}=\Delta i_{c, m}-\frac{\Delta v_{o, m}-\sum_{i=1}^{n+1} \alpha_{i} \cdot \Delta v_{o, i}}{r_{m}}\right.
\end{aligned}
$$

where $\Delta S_{c, m}, \Delta v_{p v, m}, \Delta i_{c, m}$ and $\Delta v_{o, m}$ are the corresponding offsets off the equilibrium point, $g_{p v, m}$ is the conductance of the PV panel. From (2), it can be seen that the complete and detailed model of the PV generator is nonlinear with many quadratic terms.

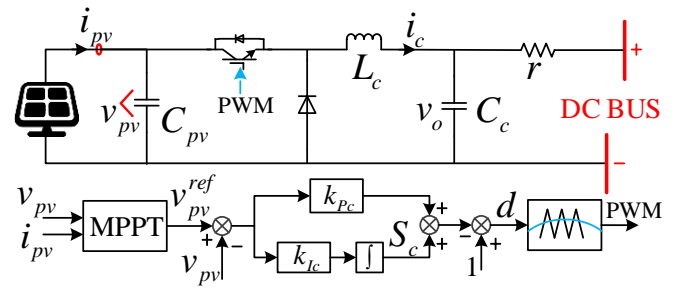

Fig.2. Structure of the $m$-th $\mathrm{PV}$ generator and its control.

\section{B. Model of the Storage}

The structure of the storage and its control is shown in Fig.3. The battery is connected to the dc bus through a bidirectional boost $\mathrm{dc} / \mathrm{dc}$ converter to boost its voltage. The filter inductance and filter capacitance are $L_{v}$ and $C_{v}$. The line resistance between the storage and the dc bus is $r$. The inductive current and the capacitive voltage on the output side are $i_{v}$ and $v_{o}$.

In the islanded dc microgrid, the dc bus voltage is maintained by the storage. Hence, the outer loop is the PI based voltage controller, which can realize the accurate voltage tracking. The inner loop is the current controller, which can realize the active damping and enhance the system stability.

Based on Fig.3, the model of the storage can be derived as

$$
\begin{aligned}
& \frac{d S_{v}}{d t}=k_{I v}\left(V_{o}^{*}-v_{o}\right) \\
& L_{v} \frac{d i_{v}}{d t}=V_{s}-\left[1-S_{v}-k_{P v}\left(V_{o}^{*}-v_{o}\right)+R_{v} i_{v}\right] v_{o}, \\
& \left(C_{v} \frac{d v_{o}}{d t}=\left[1-S_{v}-k_{P v}\left(V_{o}^{*}-v_{o}\right)+R_{v} i_{v}\right] i_{v}-\frac{v_{o}-\sum_{i=1}^{n+1} \alpha_{i} \cdot v_{o, i}}{r}\right.
\end{aligned},
$$

where $S_{v}$ is the output of the integral controller, $k_{P v}$ and $k_{I v}$ are the proportional coefficient and integral coefficient of the PI controller, $R_{v}$ is the active damping coefficient of the current controller, $V_{s}$ is the terminal voltage of the battery.

Assuming the equilibrium point is $\left(S_{v}^{*}, I_{v}^{*}, V_{o}^{*}\right)$, then (3) can be rewritten into

$$
\begin{aligned}
& \frac{d \Delta S_{v}}{d t}=-k_{I v} \Delta v_{o} \\
& L_{v} \frac{d \Delta i_{v}}{d t}=V_{o}^{*} \Delta S_{v}-R_{v} V_{o}^{*} \Delta i_{v}-\left[\left(1-S_{v}^{*}\right)+k_{P v} V_{o}^{*}+R_{v} I_{v}^{*}\right] \Delta v_{o} \\
& \quad-k_{P v} \Delta v_{o}^{2}+\Delta S_{v} \Delta v_{o}-R_{v} \Delta i_{v} \Delta v_{o} \\
& C_{v} \frac{d \Delta v_{o}}{d t}=\left[\left(1-S_{v}^{*}\right)+2 R_{v} I_{v}^{*}\right] \Delta i_{v}-I_{v}^{*} \Delta S_{v}+k_{P v} I_{v}^{*} \Delta v_{o}- \\
& \quad \frac{\Delta v_{o}-\sum_{i=1}^{n+1} \alpha_{i} \cdot \Delta v_{o, i}}{r_{n+1}}-\Delta S_{v} \Delta i_{v}+k_{P v} \Delta v_{o} \Delta i_{v}+R_{v} \Delta v_{o}^{2}
\end{aligned}
$$

where $\Delta S_{v}, \Delta i_{v}$ and $\Delta v_{o}$ are the corresponding offsets off the equilibrium point. From (4), it can be seen that the complete and detailed model of the storage is also nonlinear with many quadratic terms.

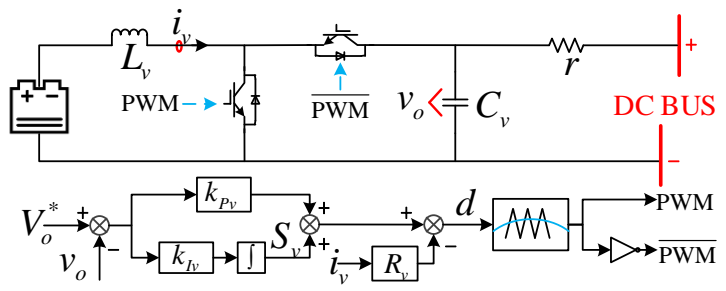

Fig.3. Structure of the storage and its control.

Choosing $\Delta S_{c 1}, \Delta v_{p v 1}, \Delta i_{c 1}, \Delta v_{o 1}, \Delta S_{c 2}, \Delta v_{p v 2}, \Delta i_{c 2}$, $\Delta v_{o 2}, \ldots, \Delta S_{v}, \Delta i_{v}, \Delta v_{o}$ as state variables $\left[x_{1}, x_{2}, \ldots, x_{n}\right]$, then based on (2) and (4), the whole model of the dc microgrid can be derived as the following compact form using matrices

$$
\dot{X}=A X+\left[X^{T} H_{1} X, X^{T} H_{2} X, \ldots, X^{T} H_{n} X\right]^{T},
$$

where $X=\left[x_{1}, x_{2}, \ldots, x_{n}\right]^{T}, H_{1}, H_{2}, \ldots, H_{n}$ are the corresponding matrices of quadratic forms. From (5), it can be concluded that the model of the dc microgrid is high-order and nonlinear. Furthermore, the nonlinearity is mainly caused by some quadratic terms.

\section{PRinciple of Nonlinear Decoupling}

This section mainly introduces the principle of the nonlinear decoupling. The proposed nonlinear decoupling is mainly based on the norm form analysis introduced by Poincare, whose detailed description can be found in [22]-[23].

\section{A. Nonlinear Transformation}

The first step called X-space to Y-space is to transform the original system (5) into its Jordan form through the following similarity transformation

$$
X=P Y,
$$

where $P$ is the matrix composed by the right eigenvectors of $A$.

Then, the original system (5) in the $\mathrm{X}$-space can be transformed into the following form in the Y-space

$$
\begin{aligned}
& \dot{Y}=\Lambda Y+P^{-1}\left[Y^{T} P^{T} H_{1} P Y, Y^{T} P^{T} H_{2} P Y, \ldots, Y^{T} P^{T} H_{n} P Y\right]^{T} \\
& =\Lambda Y+\left[Y^{T} V_{1} Y, Y^{T} V_{2} Y, \ldots, Y^{T} V_{n} Y\right]^{T} \\
& \left.\lambda_{1} y_{1}\right\rceil\left\lceil\sum_{k=1}^{n} \sum_{l=1}^{n} v_{1, k l} \cdot y_{k} y_{l}\right. \\
& =\begin{array}{llll}
\lambda_{2} y_{2} & & \\
& & \ddots & \\
& & & \left.\lambda_{n} y_{n}\right\rfloor \\
& & & \sum_{k=1}^{n} \sum_{l=1}^{n} v_{2, k l} \cdot y_{k} y_{l} \\
\vdots & \left.\sum_{k=1}^{n} \sum_{l=1}^{n} v_{n, k l} \cdot y_{k} y_{l}\right\rfloor
\end{array}
\end{aligned}
$$

where $Y=\left[y_{1}, y_{2}, \ldots, y_{n}\right]^{T}$ is the state variable vector in the Y-space, $\Lambda=P^{-1} A P=\operatorname{diag}\left\{\lambda_{1}, \lambda_{2}, \ldots, \lambda_{n}\right\}$ and $\lambda_{1}, \lambda_{2}, \ldots, \lambda_{n}$ are the eigenvalues of $A, V_{1}, V_{2}, \ldots, V_{n}$ are the corresponding matrices of quadratic forms and $V_{j}=\sum_{m=1}^{n}\left(P^{-1}\right)_{j m} \cdot\left(P^{T} H_{m} P\right)$, 
$j=1,2, \ldots, n, v_{j, k l}$ is the element of matrix $V_{j}$.

The second step called Y-space to Z-space is to transform the system (7) into a decoupled and simpler form. The wanted structure in $\mathrm{Z}$-space is that

$$
\dot{Z}=\Lambda Z+\left[Z^{T} W_{1} Z, Z^{T} W_{2} Z, \ldots, Z^{T} W_{n} Z\right]^{T}+o\left(Z^{3}\right),
$$

where $Z=\left[z_{1}, z_{2}, \ldots, z_{n}\right]^{T}$ is the state variable vector in the $Z$-space, $\Lambda$ has the same meaning as that in (7), $o\left(Z^{3}\right)$ presents the 3rd- and higher order polynomial terms, $W_{1}, W_{2}, \ldots, W_{n}$ are the corresponding matrices of quadratic forms.

Being different from the conventional norm form analysis where all quadratic nonlinear terms need to be eliminated (namely $W_{j}=0, j=1,2, \ldots, n$ ), in the proposed nonlinear decoupling, the quadratic terms do not need to be eliminated completely but they should meet the decoupling requirement.

Concretely, if $\lambda_{j}$ is a real number,

$$
\dot{z}_{j}=\lambda_{j} z_{j}+w_{j, j j} z_{j}^{2}
$$

where $z_{j}$ is the real state variable in the Z-space, $w_{j, j j}$ is the corresponding element of matrix $W_{j}$.

If $\lambda_{j}$ and $\lambda_{i}$ are a pair of conjugate complex numbers,

$$
\left\{\begin{array}{c}
\dot{z}_{j}=\lambda_{j} z_{j}+w_{j, j j} z_{j}^{2}+\left(w_{j, j i}+w_{j, i j}\right) z_{j} z_{i}+w_{j, i i} z_{i}^{2}, \\
\dot{z}_{i}=\overline{\dot{z}}_{j}
\end{array},\right.
$$

where $z_{j}$ and $z_{i}$ are the conjugate complex state variables in the Z-space, $w_{j, j j}, w_{j, j i}, w_{j, i j}$ and $w_{j, i i}$ are the corresponding elements of matrix $W_{j}$.

That is, if the third- and higher- order polynomial terms are omitted, the original nonlinear system is approximately transformed into a series of decoupled first-order quadratic or second-order quadratic systems like (9) and (10). These decoupled low-order quadratic systems retain some nonlinear nature, but they are simple enough and can be analyzed in detail, which will be introduced in next part.

To realize this goal, the following nonlinear transformation is adopted

$$
Y=Z+\left[Z^{T} T_{1} Z, Z^{T} T_{2} Z, \ldots, Z^{T} T_{n} Z\right]^{T},
$$

where $T_{1}, T_{2}, \ldots, T_{n}$ are the corresponding matrices of quadratic forms. It should be noted that this nonlinear transformation may introduce the 3rd- or higher order polynomial terms in the Z-space.

The main challenge is to solve matrices $W_{1}, W_{2}, \ldots, W_{n}$ and $T_{1}, T_{2}, \ldots, T_{n}$. If the eigenvalues of $A$ meet $\lambda_{j} \neq \lambda_{k}+\lambda_{l}$, $j, k, l=1,2, \ldots, n$, the matrices $W_{1}, W_{2}, \ldots, W_{n}$ and $T_{1}, T_{2}, \ldots$, $T_{n}$ can be calculated as follows.

For the $j$-th matrix $W_{j}, j=1,2, \ldots, n$, if $\lambda_{j}$ is a real number

$$
\left\{\begin{array}{l}
w_{j, j j}=v_{j, j j} \\
w_{j, k l}=0, \text { for others }
\end{array},\right.
$$

if $\lambda_{j}$ and $\lambda_{i}$ are a pair of conjugate complex numbers,

$$
\left\{\begin{array}{l}
w_{j, j j}=v_{j, j j}, w_{j, j i}=v_{j, j i}, w_{j, i j}=v_{j, i j}, w_{j, i i}=v_{j, i i}, \\
w_{j, k l}=0, \text { for others }
\end{array}\right.
$$

where $w_{j, k l}$ is the element of the matrix $W_{j}, v_{j, k l}$ is the element of the matrix $V_{j}$ defined in (7).

For the $j$-th matrix $T_{j}, j=1,2, \ldots, n$,

$$
t_{j, k l}=\frac{w_{j, k l}-v_{j, k l}}{\lambda_{j}-\lambda_{k}-\lambda_{l}}
$$

where $w_{j, k l}$ is the element of the matrix $W_{j}$.
Through (11)-(14), the system (7) in the Y-space can be transformed into the system (8) in the Z-space. Based on these decoupled first-order quadratic or second-order quadratic systems in the Z-space, the system stability can be analyzed effectively.

\section{B. Stability Analysis in Z-space}

The proposed nonlinear decoupling retains nonlinearity in the decoupled Z-space. Hence, some nonlinear stability issues need to be analyzed. One great difference between the nonlinear system and the linear system is that the stability of the nonlinear system is depended on the initial condition. When the initial point is in the region of attraction (ROA), the system is stable. But when the initial point is out of the ROA, then system is unstable.

For the high-order nonlinear system, the ROA evaluation is a great challenge and is still an open problem. However, for the low-order system especially for first- and second- order system, some existing results and tools can be referred to. Omitting the third- and higher- order polynomial terms, the system (8) in the Z-space is composed by a series of decoupled first- and secondorder systems. Therefore, the transient stability of system (8) can be analyzed in detail. It should be noted that the equilibrium point $Z^{*}=0$ of (8) is our interested equilibrium point and the stability of (8) around this equilibrium point is studied.

For the first-order quadratic system shown in (9), its stability analysis is relatively easy. In this paper, we mainly study the system stability under assumption that the system is small-signal stable, namely $\lambda_{j}<0$. Or else, the related unstable phenomena can be easily explained through the small-signal analysis. Under this premise, the ROA of the first-order quadratic system (9) can be derived as

$$
\left\{\begin{array}{l}
R O A=\left(-\infty,-w_{j, j j} / \lambda_{j}\right), \text { if } w_{j, j j}>0 \\
R O A=\left(-w_{j, j j} / \lambda_{j},+\infty\right), \text { if } w_{j, j j}<0
\end{array},\right.
$$

and the detailed region is depicted in Fig.4.

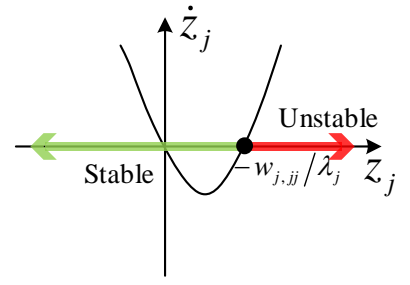

(a)

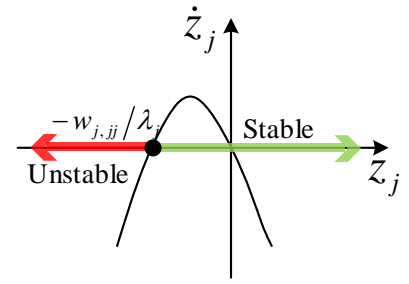

(b)
Fig.4. ROA of the first-order quadratic system. (a) $w_{j, j j}>0$. (b) $w_{j, j j}<0$.

For the second-order quadratic system shown in (10), $z_{j}$ and $z_{i}$ are conjugate, hence we only need to study the stability of $z_{j}$. For the convenient analysis, the complex expression of (10) can be divided into the real part and imaginary part, in which the related parameters are all real numbers. Then, based on the mature stability analysis tools about second-order quadratic system [24]-[26], the ROA of the second-order quadratic system (10) can be evaluated. In this paper, the trajectory reversing method based on the backward integration is adopted.

A brief overview of the trajectory reversing method is provided here, for more formal introduction, the reader can refer to [26]. For a pair of dual systems 


$$
\begin{gathered}
\dot{z}=f(z), \\
\dot{z}=-f(z),
\end{gathered}
$$

their stable region and unstable region is reverse as shown in Fig.5. That is, the stable region of (16) is the unstable region of (17), while the unstable region of (16) is the stable region of (17). According to this property, we can easily evaluate the ROA of (16) through several backward integrations and forward integrations.

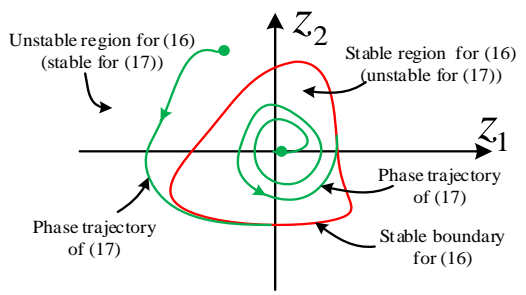

Fig.5. ROA evaluation of the second-order quadratic system.

In addition, the initial condition in the Z-space should be calculated out. Due to the nonlinear transformation in (11), calculating $Z_{0}$ according to $X_{0}$ needs to solve some nonlinear equations. Then, the following procedures are adopted to obtain the initial condition $Z_{0}$ in the Z-space after knowing $X_{0}$.

1) Calculating $Y_{0}: Y_{0}=P^{-1} X_{0}, P$ is defined in (6).

2) Calculating $Z_{0}: Z_{0}$ can be obtained through solving roots of the equation $Z+\left[Z^{T} T_{1} Z, Z^{T} T_{2} Z, \ldots, Z^{T} T_{n} Z\right]^{T}-Y_{0}=0$. This equation can be iteratively solved out by using Newton-Raphson method with the initial value $Z=Y_{0}$.

When the ROAs and the initial condition are determined, the stability of these decoupled first-order or second-order quadratic systems in the Z-space can be analyzed. Then, the stability of the original system (5) can be reflected. Also, from the above procedures, it can be seen that the proposed nonlinear decoupling based analysis framework is not limited to the system orders. Hence, compared to the Lyapunov based stability analysis methods, the proposed method can provide more accurate and less conservative results of transient stability for the high-order nonlinear system.

\section{CASE STUdies}

To verify the effectiveness of the proposed nonlinear decoupling based analysis framework, a 3-machine $\mathrm{dc}$ microgrid system has been studied, whose topology is shown in Fig.6. Concretely, on the source side, there are two PV generators provide power support and one storage to maintain the dc bus voltage. On the load side, the system load is presented by a lumped resistor $R_{L}$.

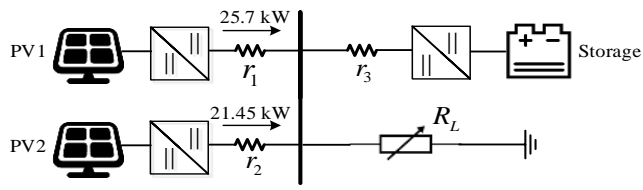

Fig.6. Studied 3-machine dc microgrid system.

The rated dc bus voltage is $300 \mathrm{~V}$ and the rated load $R_{L}=$ $1 \Omega$. With different test cases, the dc bus voltage and the load will change. Hence, these two variables are used to present different states. The used PV module in this paper is KC200GT, whose detailed parameters are introduced in [27]. The PV panel of PV1 consists of 20 series and 6 parallel modules, while the PV panel of PV2 consists of 20 series and 5 parallel modules. The tested irradiance and temperature are $1000 \mathrm{~W} / \mathrm{m}^{2}$ and 298.16 K respectively. The PV generators and storage are under the control introduced in Section II, and their parameters are shown in Table I and Table II.

TABLE I

\begin{tabular}{|c|c|c|}
\hline \multirow{2}{*}{ Parameters } & \multicolumn{2}{|c|}{ Rated Value } \\
\hline & PV1 & PV2 \\
\hline $\begin{array}{c}\text { Filters } \\
\left(C_{p v}, L_{c}, L_{c}\right)\end{array}$ & $0.3 \mathrm{mF}, 1.5 \mathrm{mH}, 0.5 \mathrm{mF}$ & $0.5 \mathrm{mF}, 3 \mathrm{mH}, 0.8 \mathrm{mF}$ \\
\hline Line Resistance & $r_{1}=200 \mathrm{~m} \Omega$ & $r_{2}=100 \mathrm{~m} \Omega$ \\
\hline Rated Power & $25.7 \mathrm{~kW}$ & $21.45 \mathrm{~kW}$ \\
\hline PV Conductance & $g_{p v 1}=-0.0686 \mathrm{~A} / \mathrm{V}$ & $g_{p v 2}=-0.0572 \mathrm{~A} / \mathrm{V}$ \\
\hline $\begin{array}{c}\text { Controller } \\
\left(k_{P c}, k_{I c}\right)\end{array}$ & $0.02,1$ & $0.05,0.5$ \\
\hline
\end{tabular}

PARAMETERS OF PV GENERATORS

\section{A. Case 1: Voltage Changes}

Usually, the dc bus voltage will change in a reasonable range (like $\pm 10 \%$ ) to realize some coordination function like generation-storage coordination, ac/dc microgrids coordination and so on [28]-[29]. In this case, we mainly study the transient stability introduced by the voltage changes.

TABLE II

PARAMETERS OF THE STORAGE

\begin{tabular}{cc}
\hline \hline Parameters & Rated Value \\
\hline Filters & $L_{v}=3 \mathrm{mH}, C_{v}=1.5 \mathrm{mF}$ \\
Line Resistance & $r_{3}=200 \mathrm{~m} \Omega$ \\
Reference Voltage & $V_{o}^{*}=300 \mathrm{~V}$ \\
Battery Voltage & $V_{s}=100 \mathrm{~V}$ \\
Controller & $k_{P v}=0.01, k_{I v}=8.5, R_{v}=0.02$ \\
\hline \hline
\end{tabular}

Three small-signal stable states $(300 \mathrm{~V}, 1 \Omega),(295 \mathrm{~V}, 1 \Omega)$, and $(285 \mathrm{~V}, 1 \Omega)$ are chosen to conduct the test, whose system poles after linearization is shown in Fig.7. Concretely, the states $(295 \mathrm{~V}, 1 \Omega)$ and $(285 \mathrm{~V}, 1 \Omega)$ are view as the initial states, while the state $(300 \mathrm{~V}, 1 \Omega)$ is viewed as final state and the transient stability is analyzed during the system jumps to this state from the above two different initial states. That is, the steady system state variables when the dc microgrid operates in the state $(300 \mathrm{~V}, 1 \Omega)$ constitutes the equilibrium point, while the steady system state variables when the dc microgrid operates in the states $(295 \mathrm{~V}, 1 \Omega)$ and $(285 \mathrm{~V}, 1 \Omega)$ constitutes the initial points.

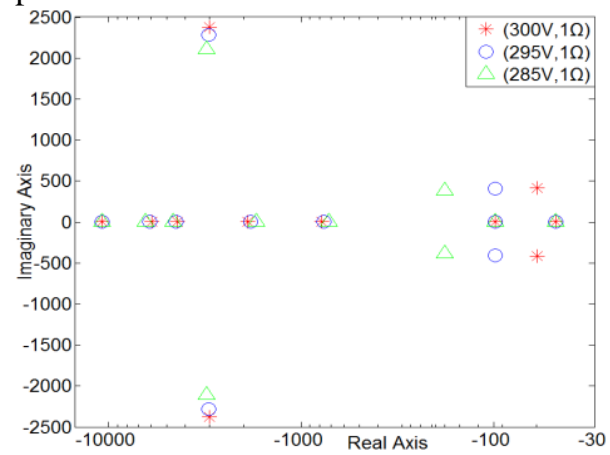

Fig.7. System poles under different dc bus voltages after linearization.

From Fig.7, it can be seen that the small-signal stability of these three states has no obvious difference. Especially, they almost have the same dominated poles with enough stable 
margin. Hence, only through the small-signal analysis, the system transient stability during the transition among these states cannot be reflected effectively.

According to the introduced procedures about the nonlinear decoupling in section III, the decoupled state equation of the dc microgrid in the equilibrium state $(300 \mathrm{~V}, 1 \Omega)$ can be derived as

$$
\begin{aligned}
\dot{z}_{1}= & -10833 z_{1}-0.0051 z_{1}^{2} \\
\dot{z}_{2}= & -5963 z_{2}-96.0525 z_{2}^{2} \\
\dot{z}_{3}= & -4398 z_{3}-1.3982 z_{3}^{2} \\
\dot{z}_{4}= & (-2988+j 2377) z_{4}-(26.2118+j 34.1158) z_{4}^{2}+ \\
& (3.1625-j 67.5694) z_{4} z_{5}+(27.5359-j 31.1786) z_{5}^{2} \\
\dot{z}_{5}= & \dot{z}_{4} \\
\dot{z}_{6}= & -1894 z_{6}-28.2769 z_{6}^{2} \\
\dot{z}_{7}= & -779 z_{7}+3.488 z_{7}^{2} \\
\dot{z}_{8}= & (-60+j 412) z_{8}+(1.0556+j 1.6098) z_{8}^{2}- \\
& (0.2816+j 1.3592) z_{8} z_{9}+(-0.7145+j 1.3517) z_{9}^{2} \\
\dot{z}_{9}= & \dot{z}_{8} \\
\dot{z}_{10}= & -98 z_{10}+0.0034 z_{10}^{2} \\
\dot{z}_{11}= & -48 z_{11}+0.0039 z_{11}^{2}
\end{aligned}
$$

Also, the two initial points can be obtained according to the introduced procedures about calculating initial conditions in Section III, and the corresponding results are shown in Table III. From the table, it can be seen that the initial values of $z_{8}$ and $z_{9}$ are relatively larger, which are easy to exceed the ROA and cause transient instability. Hence, the second-order quadratic system comprised of $z_{8}$ and $z_{9}$ should be paid more attention. Since $z_{8}$ and $z_{9}$ are conjugate, only the dynamics of $z_{8}$ need to be studied. For the convenient analysis, the corresponding complex differential equation about $z_{8}$ is divided into the real part and imaginary part as follows

$$
\left[\begin{array}{c}
\dot{z}_{R} \\
\dot{z}_{I}
\end{array}\right]=\left[\begin{array}{cc}
-60 & -412 \\
412 & -60
\end{array}\right]\left[\begin{array}{l}
z_{R} \\
z_{I}
\end{array}\right]+\left[\begin{array}{c}
{\left[z_{R}, z_{I}\right]\left[\begin{array}{cc}
0.0595 & -0.2581 \\
-0.2581 & -0.6227
\end{array}\right]\left[\begin{array}{c}
z_{R} \\
z_{I}
\end{array}\right]} \\
{\left[z_{R}, z_{I}\right]\left[\begin{array}{cc}
1.6023 & 1.7701 \\
1.7701 & -4.3207
\end{array}\right]\left[\begin{array}{c}
Z_{R} \\
z_{I}
\end{array}\right]}
\end{array}\right],
$$

where $z_{8}=z_{R}+j z_{I}, z_{9}=z_{R}-j z_{I}$.

$$
\text { TABLE III }
$$

INITIAL CONDITIONS RELATIVE TO THE STATE $(300 \mathrm{~V}, 1 \Omega)$

\begin{tabular}{cc}
\hline \hline Initial States & Initial Points $Z_{0}$ \\
\hline \multirow{2}{*}{$(295 \mathrm{~V}, 1 \Omega)$} & $(-0.3076,0.2597,1.3924,0.0653-\mathrm{j} 0.3368,0.0653+\mathrm{j} 0.3368$ \\
& $,-11.0120,2.4078, \mathbf{- 1 8 . 4 1 8 9 - j 7 . 4 8 1 9 , - 1 8 . 4 1 8 9 + j 7 . 4 8 1 9 ,}$ \\
\hline \multirow{2}{*}{$(285 \mathrm{~V}, 1 \Omega)$} & $(0.0858,0.0195,-2.5209,0.0581-\mathrm{j} 0.4326,0.0581+\mathrm{j} 0.4326$ \\
& $, 3.8570,3.8831,-\mathbf{5 6 . 7 1 5 7 - j 2 4 . 0 5 6 3 , - 5 6 . 7 1 5 7 + j 2 4 . 0 5 6 3 ,}$ \\
\hline \hline
\end{tabular}

Based on (19) and Table III, the transient stability of the dc microgrid under the changes of the dc bus voltage can be analyzed. Fig.8 (a) shows the evaluated ROA of $z_{8}$ in the equilibrium state $(300 \mathrm{~V}, 1 \Omega)$. Through this figure, it can be found that the initial state $(295 \mathrm{~V}, 1 \Omega)$ is in the ROA, while the initial state $(285 \mathrm{~V}, 1 \Omega)$ is out of the ROA. Hence, the dc microgrid can keep stable when it jumps from the state $(295 \mathrm{~V}$, $1 \Omega)$ to the state $(300 \mathrm{~V}, 1 \Omega)$, whose phase trajectory is convergent to zero (namely the equilibrium point) and is shown in Fig.8 (b). In contrast, the dc microgrid cannot keep stable when it jumps from the state $(285 \mathrm{~V}, 1 \Omega)$ to the state $(300 \mathrm{~V}$, $1 \Omega$ ), whose phase trajectory is divergent and is shown in Fig.8 (c). That is, the dc microgrid cannot reach the state $(300 \mathrm{~V}, 1 \Omega)$ directly from the state $(285 \mathrm{~V}, 1 \Omega)$. Through Fig.8, the transient stability of the dc microgrid under the voltage changes can be reflected clearly.

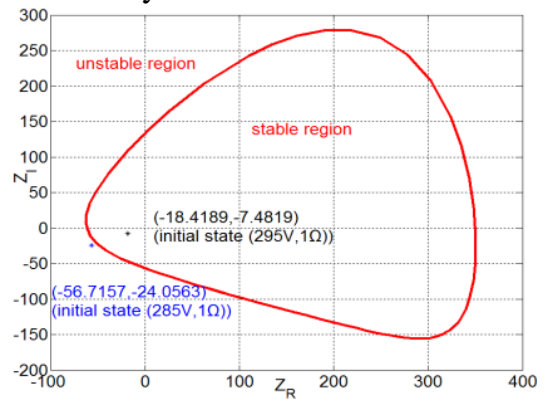

(a)

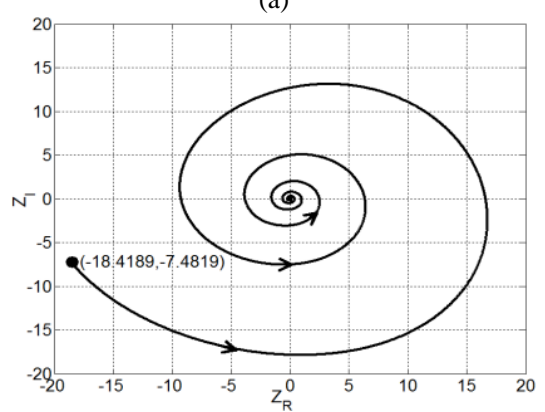

(b)

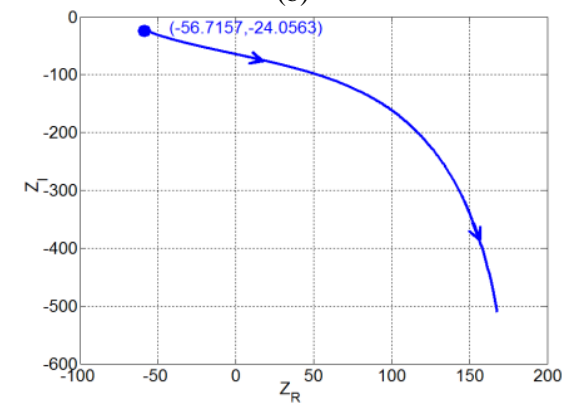

(c)

Fig.8. Transient stability of $z_{8}$ in (19). (a) ROA. (b) Phase trajectory of the initial point (-18.4189,-7.4819) (belongs to $(295 \mathrm{~V}, 1 \Omega)$ ). (c) Phase trajectory of the initial point $(-56.7157,-24.0563)$ (belongs to $(285 \mathrm{~V}, 1 \Omega)$ ).

To further verify the correctness of the proposed analysis method, the related time-domain simulation is conducted and the corresponding results are shown in Fig.9.

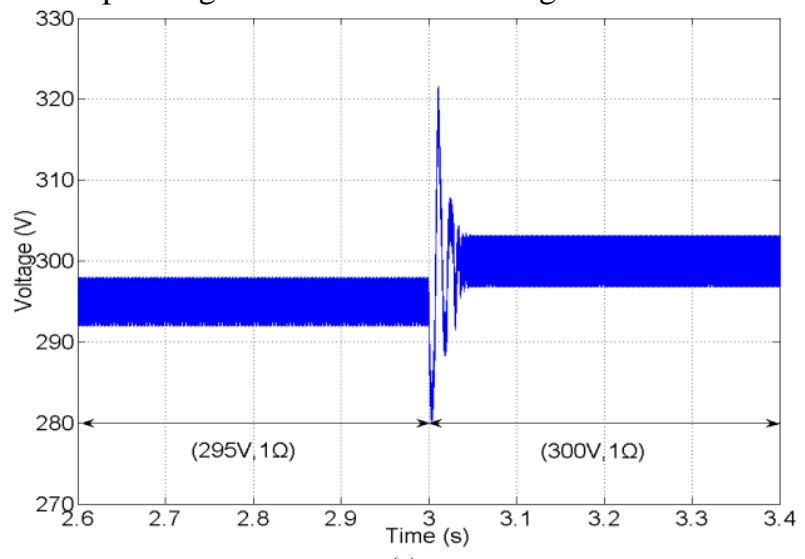

(a) 


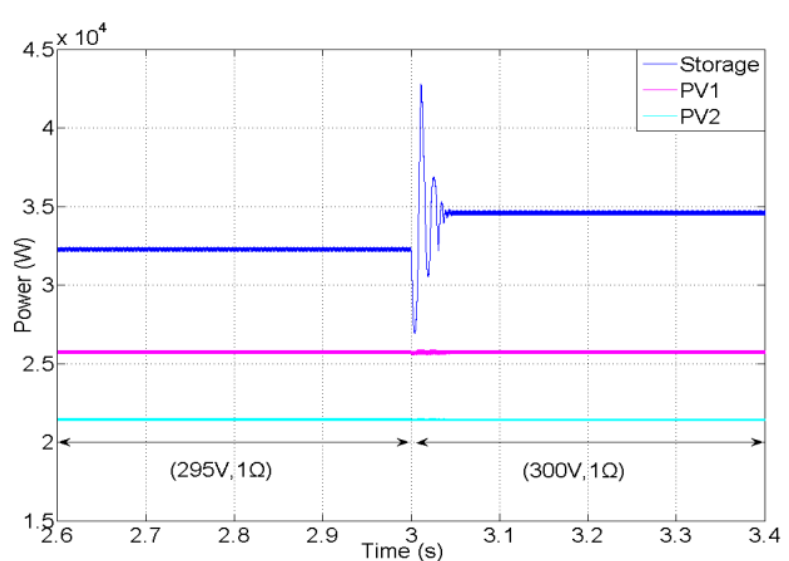

(b)

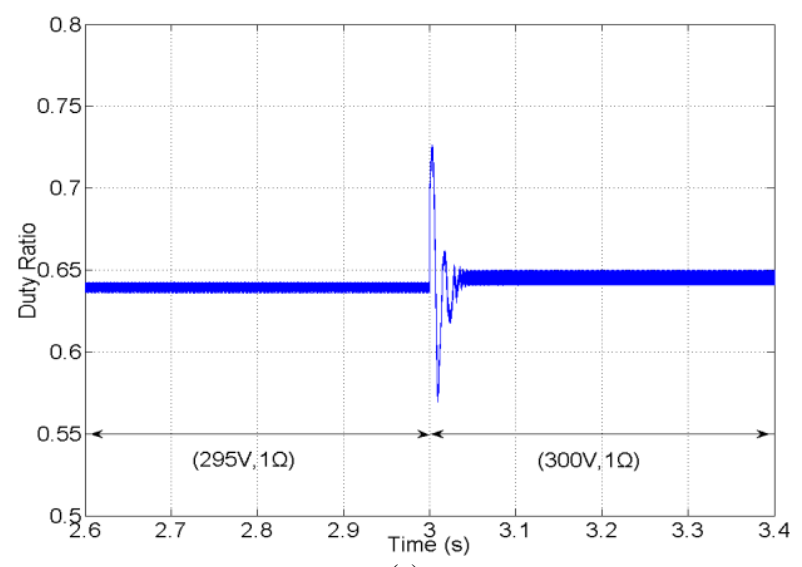

(c)

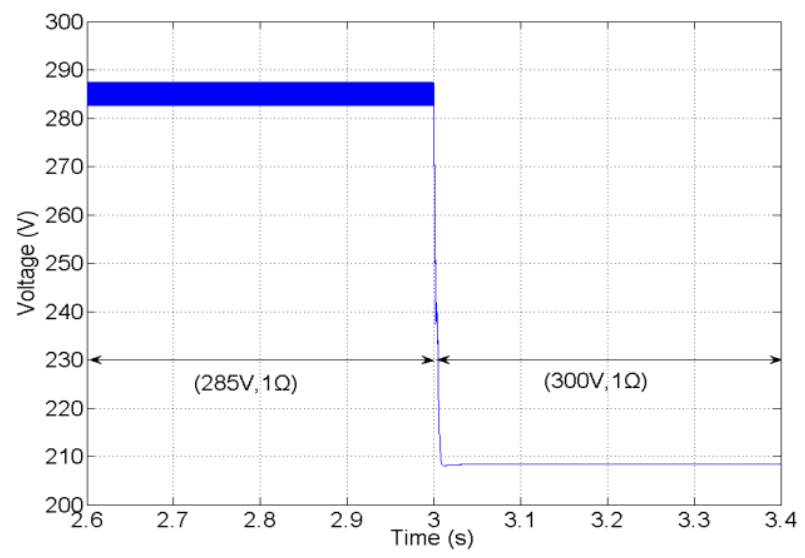

(d)

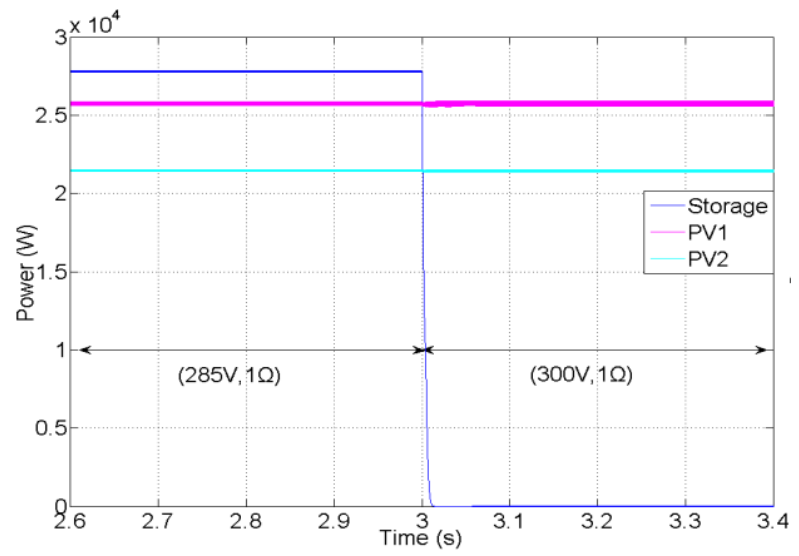

(e)

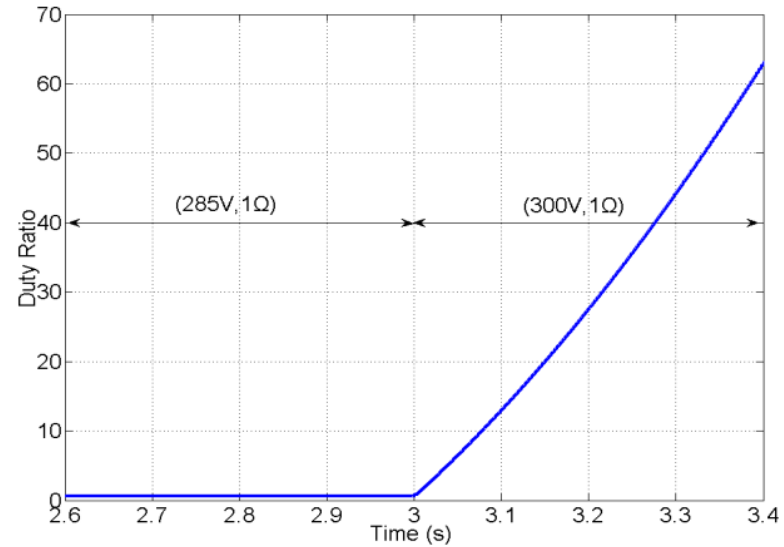

(f)

Fig.9. System dynamics during the states transition. Stable transition from the state $(295 \mathrm{~V}, 1 \Omega)$ to the state $(300 \mathrm{~V}, 1 \Omega)$. (a) Output voltage of the storage. (b) Output power of three sources. (c) Duty ratio of the storage converter. Unstable transition from the state $(285 \mathrm{~V}, 1 \Omega)$ to the state $(300 \mathrm{~V}, 1 \Omega)$. (d) Output voltage of the storage. (e) Output power of three sources. (f) Duty ratio of the storage converter

Through the time-domain simulation results, it can be seen that for the two closer states $(295 \mathrm{~V}, 1 \Omega)$ and $(300 \mathrm{~V}, 1 \Omega)$, their transition can be stabilized and the system can reach the state $(300 \mathrm{~V}, 1 \Omega)$ from the state $(295 \mathrm{~V}, 1 \Omega)$. As shown in Fig.9 (a) and (b), both the output voltage and output power of the storage can follow the changes in time. At the same time, the duty ratio used to control the storage converter is within the allowed change range $[0,1]$ as Fig.9 (c) shows.

But for the two farther states $(285 \mathrm{~V}, 1 \Omega)$ and $(300 \mathrm{~V}, 1 \Omega)$, their transition is unstable. That is, from the stable state $(285 \mathrm{~V}$, $1 \Omega)$, the system cannot reach another stable state $(300 \mathrm{~V}, 1 \Omega)$ directly. During the transition, the storage cannot maintain the dc bus voltage within the allowed range as Fig.9 (d) shows. Consequently, the storage becomes unstable and is blocked, then its output power decreases to zero as Fig.9 (e) shows. Also, in Fig.10 (f), it can be seen that the duty ratio far exceeds the allowed change range $[0,1]$.

The results of time-domain simulation meet the theoretical analysis in Fig.8 well. It is also revealed that for the small-signal stable states, their transition may be unstable.

\section{B. Case2: Load Changes}

In reality, the dc microgrid suffers the uncertainty of loads, which will influence the system stability. Therefore, in this case, we mainly study the transient stability introduced by the load changes.

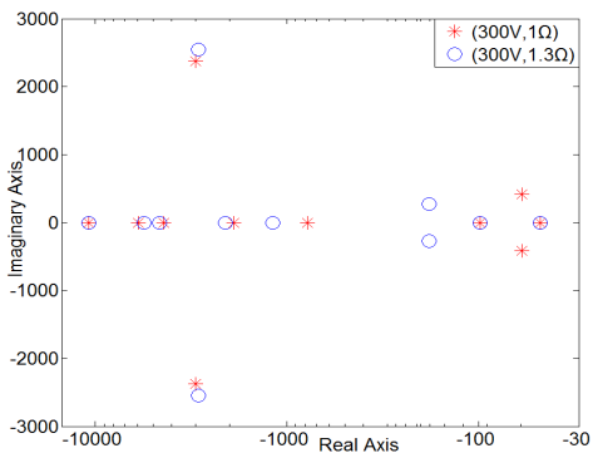

Fig.10. System poles under different loads after linearization.

Two small-signal stable states $(300 \mathrm{~V}, 1 \Omega)$ and $(300 \mathrm{~V}$, 
$1.3 \Omega$ ) are chosen to conduct the test, whose system poles after linearization is shown in Fig.10. Their mutual transition is mainly studied, that is the transient stability is analyzed when the dc microgrid jumps from the state $(300 \mathrm{~V}, 1.3 \Omega)$ to the state $(300 \mathrm{~V}, 1 \Omega)$ and its inverse process.

Being similar to Fig.7, Fig.10 shows that the system small-signal stability under these two different loads has no obvious difference. That is, the system transient stability during their mutual transition cannot be obtained effectively only through the simple linear analysis.

The decoupled state equation of the dc microgrid in the equilibrium state $(300 \mathrm{~V}, 1 \Omega)$ has been derived in (18). Now, the decoupled state equation of the dc microgrid in the equilibrium state ( $300 \mathrm{~V}, 1.3 \Omega$ ) needs to be derived, which is shown in (20). And the initial points of different states are shown in Table IV.

$$
\begin{aligned}
& \dot{z}_{1}=-10833 z_{1}+0.0049 z_{1}^{2} \\
& \dot{z}_{2}=(-2903+j 2550) z_{2}+(28.1373+j 32.4023) z_{2}^{2}+ \\
& (-2.7524+j 64.6582) z_{2} z_{3}+(-28.6585+j 29.7081) z_{3}^{2} \\
& \dot{z}_{3}=\dot{\bar{z}}_{2} \\
& \dot{z}_{4}=-5560 z_{4}+78.8424 z_{4}^{2} \\
& \dot{z}_{5}=-4606 z_{5}-7.0704 z_{5}^{2} \\
& \dot{z}_{6}=-2088 z_{6}+35.6246 z_{6}^{2} \\
& \dot{z}_{7}=-1186 z_{7}+8.3818 z_{7}^{2} \\
& \dot{z}_{8}=(-181+j 273) z_{8}+(0.0951+j 0.6206) z_{8}^{2}+ \\
& \quad(-0.1382+j 0.6118) z_{8} z_{9}+(-0.3447+j 0.4198) z_{9}^{2} \\
& \dot{z}_{9}=\dot{z}_{8} \\
& \dot{z}_{10}=-98 z_{10}+0.0033 z_{10}^{2} \\
& \dot{z}_{11}=-48 z_{11}+0.0040 z_{11}^{2}
\end{aligned}
$$

From the table, it can be seen that for both equilibrium states, the initial values of the conjugate $z_{8}$ and $z_{9}$ are relatively larger,

\begin{tabular}{|c|c|}
\hline Initial States & Initial Points $Z_{0}$ \\
\hline $\begin{array}{l}(300 \mathrm{~V}, 1 \Omega) \\
(\text { relative to } \\
(300 \mathrm{~V}, 1.3 \Omega))\end{array}$ & 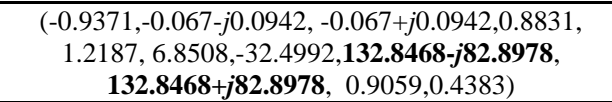 \\
\hline $\begin{array}{l}(300 \mathrm{~V}, 1.3 \Omega) \\
(\text { relative to } \\
(300 \mathrm{~V}, 1 \Omega))\end{array}$ & $\begin{array}{c}(-0.4883,0.6631,5.954,0.1872+j 0.3627 \\
0.1872-j 0.3627,-13.0311,132.9666,-\mathbf{1 4 1 . 3 7 7 4 - j 5 . 7 8 9 4} \\
, \quad \mathbf{- 1 4 1 . 3 7 7 4 + j 5 . 7 8 9 4 ,} 2.1828,0.1688) \\
\end{array}$ \\
\hline
\end{tabular}
which are easy to exceed the ROA and cause transient instability. Hence, the second-order quadratic system comprised of $z_{8}$ and $z_{9}$ should be studied. For the state (300 V, $1 \Omega$ ), the differential equation about $z_{8}$ is derived in (19) based on the divided real part and imaginary part. For the state ( $300 \mathrm{~V}$, $1.3 \Omega$ ), the corresponding complex differential equation about $z_{8}$ can be divided into the real part and imaginary part as

$$
\left[\begin{array}{c}
\dot{z}_{R} \\
\dot{z}_{I}
\end{array}\right]=\left[\begin{array}{cc}
-197 & -218 \\
218 & -197
\end{array}\right]\left[\begin{array}{c}
Z_{R} \\
z_{I}
\end{array}\right]+\left[\begin{array}{c}
{\left[z_{R}, z_{I}\right]\left[\begin{array}{cc}
-0.4014 & -0.1558 \\
-0.1558 & 0.0354
\end{array}\right]\left[\begin{array}{c}
Z_{R} \\
z_{I}
\end{array}\right]} \\
{\left[z_{R}, z_{I}\right]\left[\begin{array}{ccc}
1.7282 & 0.6986 \\
0.6986 & 0.2474
\end{array}\right]\left[\begin{array}{c}
Z_{R} \\
z_{I}
\end{array}\right]}
\end{array}\right],
$$

where $z_{8}=z_{R}+j z_{I}, z_{9}=z_{R}-j z_{I}$.

$$
\text { TABLE IV }
$$

INITIAL CONDITIONS OF DIFFERENT STATES

Based on (19), (21) and Table IV, the transient stability of the dc microgrid under the changes of loads can be analyzed. Fig.11 (a) shows the evaluated ROA of $z_{8}$ in the equilibrium state $(300 \mathrm{~V}, 1.3 \Omega$ ).Through this figure, it can be found that the initial state $(300 \mathrm{~V}, 1 \Omega)$ is in the ROA. Hence, the dc microgrid can keep stable when it jumps from the state $(300 \mathrm{~V}$, $1 \Omega)$ to the state $(300 \mathrm{~V}, 1.3 \Omega)$, whose phase trajectory is convergent to zero (namely the equilibrium point) and is shown in Fig.11(b).

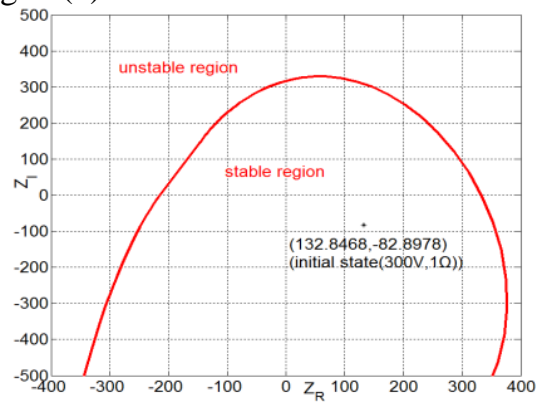

(a)

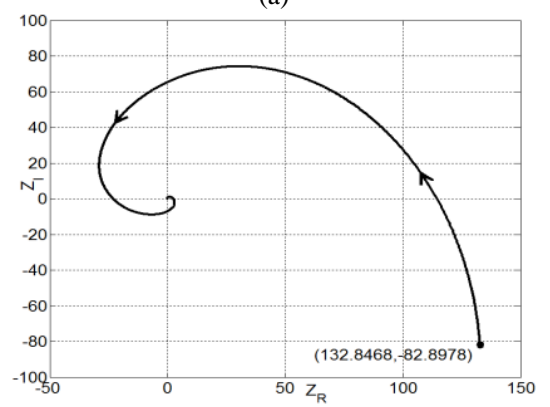

(b)

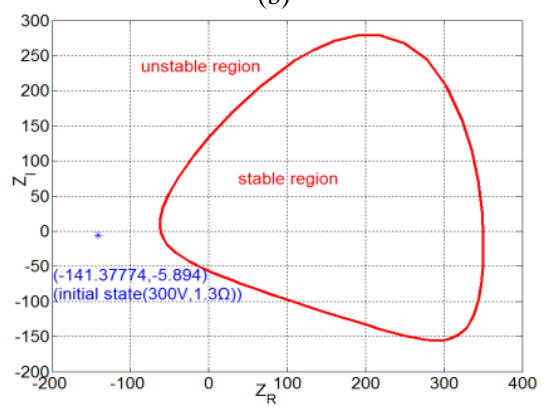

(c)

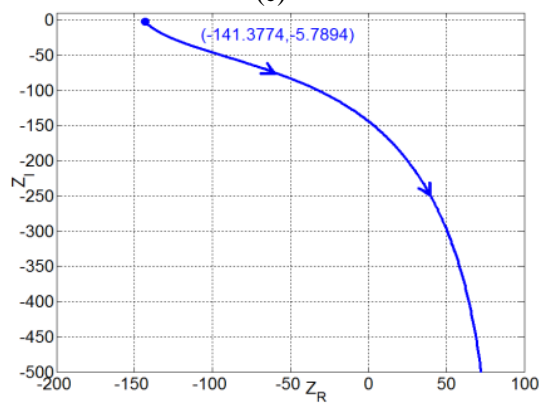

(d)

Fig.11. Transient stability of $z_{8}$ in (19) and (21). (a) ROA of the state (300 V, $1.3 \Omega$ ). (b) Phase trajectory of the initial point (-193.1882,-191.6823) (belongs to $(300 \mathrm{~V}, 1 \Omega))$. (c) ROA of the state $(300 \mathrm{~V}, 1 \Omega)$. (b) Phase trajectory of the initial point (-141.3774,-5.7894) (belongs to $(300 \mathrm{~V}, 1.3 \Omega)$ ).

However its inverse process is unstable, that is, the dc microgrid cannot keep stable when it jumps from the state (300 $\mathrm{V}, 1.3 \Omega$ ) to the state $(300 \mathrm{~V}, 1 \Omega)$. As shown in Fig.11 (c), the initial state is out of the evaluated ROA of $z_{8}$ in the equilibrium state $(300 \mathrm{~V}, 1 \Omega)$, which means that this transition is transient unstable. The corresponding phase trajectory is divergent and is 
shown in Fig.11 (d). Through Fig.11, the transient stability of the dc microgrid under the load changes can be reflected clearly.

To further verify the correctness of the proposed analysis method, the related time-domain simulation is conducted and the corresponding results are shown in Fig.12.

Through the time-domain simulation results, it can be seen that when the dc microgrid jumps from the state $(300 \mathrm{~V}, 1 \Omega)$ to the state $(300 \mathrm{~V}, 1.3 \Omega)$, the system can keep stable. As shown in Fig.12 (a) and (b), both the output voltage and output power of the storage can follow the changes in time but undergo great overshoot. Also, the duty ratio used to control the storage converter is within the allowed change range $[0,1]$ as Fig.12 (c) shows.

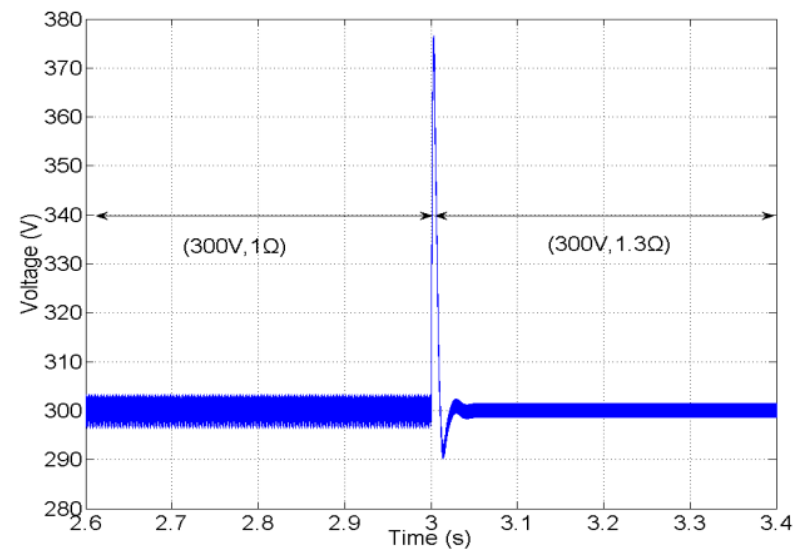

(a)

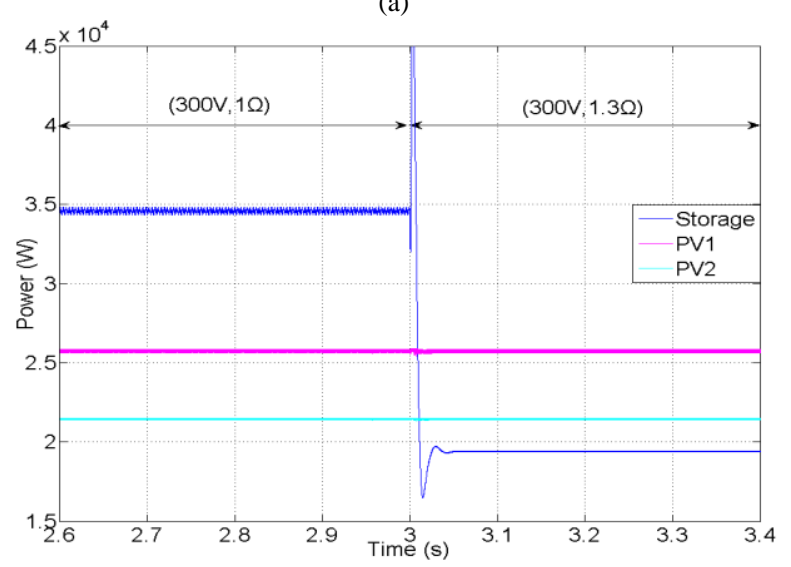

(b)

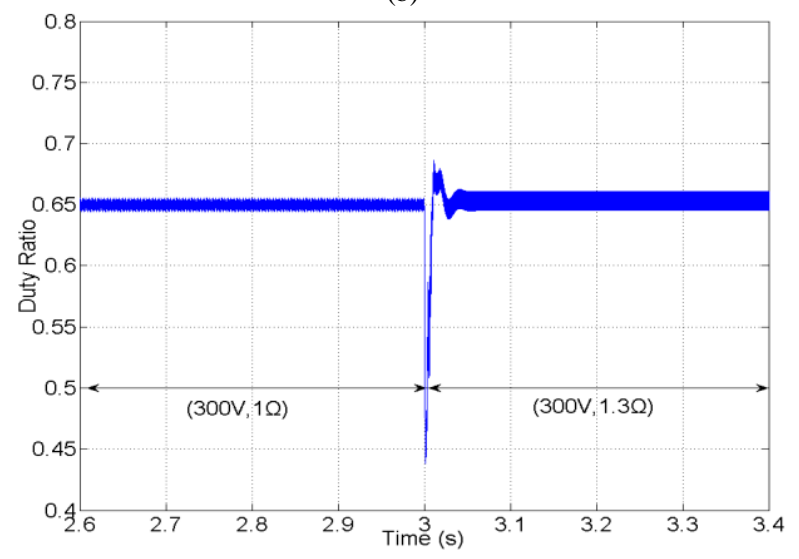

(c)

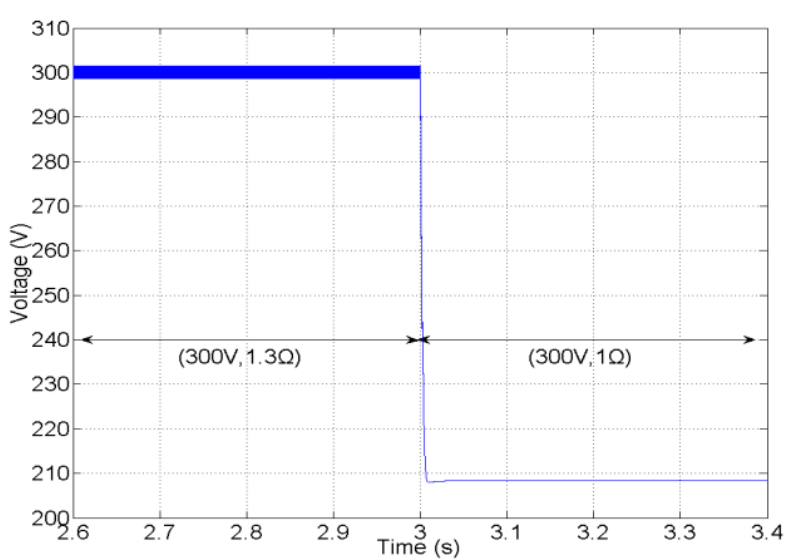

(d)

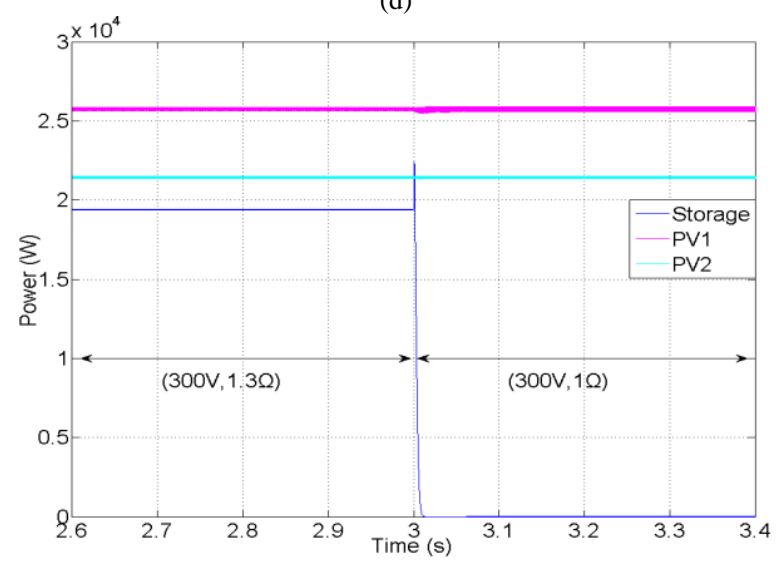

(e)

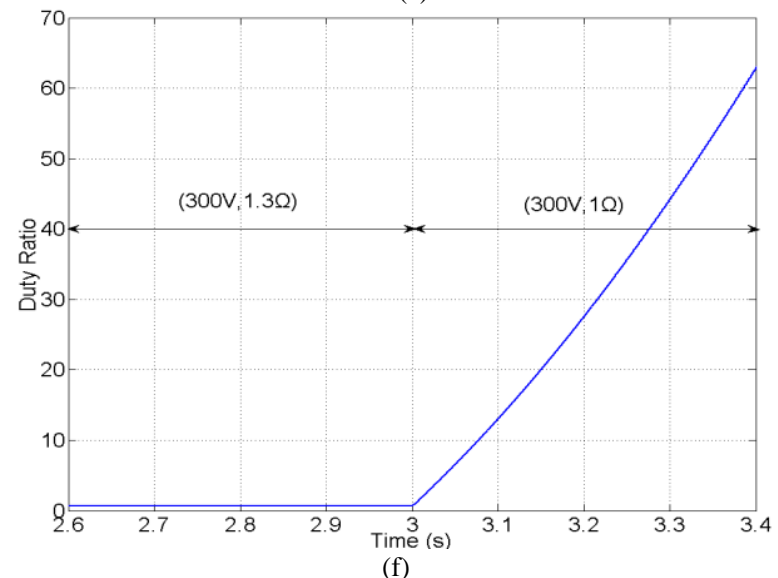

Fig.12. System dynamics during the states transition. Stable transition from the state $(300 \mathrm{~V}, 1 \Omega)$ to the state $(300 \mathrm{~V}, 1.3 \Omega)$. (a) Output voltage of the storage. (b) Output power of three sources. (c) Duty ratio of the storage converter. Unstable transition from the state $(300 \mathrm{~V}, 1.3 \Omega)$ to the state $(300 \mathrm{~V}, 1 \Omega)$. (d) Output voltage of the storage. (e) Output power of three sources. (f) Duty ratio of the storage converter

However, when the dc microgrid jumps from the state $(300 \mathrm{~V}$, $1.3 \Omega$ ) to the state $(300 \mathrm{~V}, 1 \Omega)$, the system is unstable. During the transition, the storage cannot maintain the dc bus voltage anymore and the dc bus voltage is out of the allowed change range as Fig.12 (d) shows. As a result, the storage becomes unstable and is blocked, then its output power decreases to zero as Fig.12 (e) shows. Also, the corresponding duty ratio far exceeds the allowed change range $[0,1]$ as shown in Fig.12 (f).

The results of time-domain simulation meet the theoretical analysis in Fig.11 well. At the same time, it is an interesting phenomenon that in some stressed states of the dc microgrid, 
the system dynamics of adding and cutting same large loads is not invertible. One of them may cause the system instability.

\section{CONCLUSION}

This paper proposes a nonlinear decoupling based analysis framework to study the transient stability of dc microgrids. In the proposed framework, the nonlinearity of the dc microgrid system is fully considered and the original nonlinear system is approximately transformed into a series of decoupled first-order quadratic or second-order quadratic systems, whose dynamics and stability can be analyzed easily. Then, the transient stability of the original system can be reflected indirectly. The proposed analysis framework can overcome high-order and nonlinear challenges effectively, which is very suitable for the transient stability evaluation of dc microgrids. Through the case studies, the accuracy of the proposed analysis method is further verified. At the same time, some interesting phenomena can be explained well by the proposed method. Also, the nonlinear decoupling based analysis framework can be extended to other power electronics dominated power systems to evaluate their transient stability.

\section{REFERENCES}

[1] Q. Xu, C. Zhang, C. Wen and P. Wang, "A Novel Composite Nonlinear Controller for Stabilization of Constant Power Load in DC Microgrid," IEEE Trans. Smart Grid, vol. 10, no. 1, pp. 752-761, Jan. 2019.

[2] Y. Xia, W. Wei, Y. Peng, P. Yang and M. Yu, "Decentralized Coordination Control for Parallel Bidirectional Power Converters in a Grid-Connected DC Microgrid," IEEE Trans. Smart Grid, vol. 9, no. 6, pp. 6850-6861, Nov. 2018.

[3] L. Guo, S. Zhang, X. Li, Y. W. Li, C. Wang and Y. Feng, "Stability Analysis and Damping Enhancement Based on Frequency-Dependent Virtual Impedance for DC Microgrids," IEEE J. Emerg. Sel. Topics Power Electron., vol. 5, no. 1, pp. 338-350, Mar. 2017.

[4] Y. Xia, W. Wei, M. Yu and P. Wang, "Stability Analysis of PV Generators With Consideration of P\&O-Based Power Control," IEEE Trans. Ind. Electron., vol. 66, no. 8, pp. 6483-6492, Aug. 2019.

[5] X. Lu, K. Sun, J. M. Guerrero, J. C. Vasquez, L. Huang and J. Wang, "Stability Enhancement Based on Virtual Impedance for DC Microgrids With Constant Power Loads," IEEE Trans. Smart Grid, vol. 6, no. 6, pp. 2770-2783, Nov. 2015.

[6] K. Mehran, D. Giaouris, and B. Zahawi, "Stability analysis and control of nonlinear phenomena in boost converters using model-based TakagiSugeno fuzzy approach, " IEEE Trans. Circuits Syst. I: Reg. Papers, vol. 57, no. 1, pp. 200-212, Jan. 2010.

[7] Z. Li, W. Pei, H. Ye and L. Kong, "Large signal stability analysis for DC microgrid under droop control based on mixed potential theory," J. Eng., vol. 2019, no. 16, pp. 1189-1193, Mar. 2019.

[8] D. Peng, M. Huang, J. Li, J. Sun, X. Zha and C. Wang, "Large-Signal Stability Criterion for Parallel-Connected DC-DC Converters with Current Source Equivalence," IEEE Trans. Circuits Syst. II, Exp. Briefs, to be published, doi: 10.1109/TCSII.2019.2895842.

[9] J. Jiang, F. Liu, S. Pan, X. Zha, W. Liu, C. Chen and L. Hao, "A Conservatism-Free Large Signal Stability Analysis Method for DC Microgrid Based on Mixed Potential Theory," IEEE Trans. Power Electron., to be published, doi: 10.1109/TPEL.2019.2897643.
[10] B. P. Loop, S. D. Sudhoff, S. H. Zak, and E. L. Zivi, "Estimating regions of asymptotic stability of power electronics systems using genetic algorithms, " IEEE Trans. Control Syst. Technol., vol. 18, no. 5, pp. 10111022, Sep. 2010.

[11] C. J. Sullivan, S. D. Sudhoff, E. L. Zivi, and S. H. Zak, "Methods of optimal Lyapunov function generation with application to power electronic converters and systems, " in Proc. IEEE Electric Ship Technol. Symp., 2007, pp. 267-274.

[12] L. Herrera, W. Zhang and J. Wang, "Stability Analysis and Controller Design of DC Microgrids With Constant Power Loads," IEEE Trans. Smart Grid, vol. 8, no. 2, pp. 881-888, March 2017.

[13] J. Liu, W. Zhang and G. Rizzoni, "Robust Stability Analysis of DC Microgrids With Constant Power Loads," IEEE Trans. Power Syst., vol. 33, no. 1, pp. 851-860, Jan. 2018.

[14] D. Marx, P. Magne, B. Nahid-Mobarakeh, S. Pierfederici and B. Davat, "Large Signal Stability Analysis Tools in DC Power Systems With Constant Power Loads and Variable Power Loads-A Review," IEEE Trans. Power Electron., vol. 27, no. 4, pp. 1773-1787, April 2012.

[15] M. Kabalan, P. Singh and D. Niebur, "Large Signal Lyapunov-Based Stability Studies in Microgrids: A Review," IEEE Trans. Smart Grid, vol. 8, no. 5, pp. 2287-2295, Sept. 2017.

[16] N. Kshatriya, U. D. Annakkage, A. M. Gole and I. T. Fernando, "Improving the accuracy of normal form analysis," IEEE Trans. Power Syst., vol. 20, no. 1, pp. 286-293, Feb. 2005.

[17] J. J. Sanchez-Gasca et al., "Inclusion of higher order terms for small-signal (modal) analysis: committee report-task force on assessing the need to include higher order terms for small-signal (modal) analysis," IEEE Trans. Power Syst., vol. 20, no. 4, pp. 1886-1904, Nov. 2005.

[18] T. Tian, X. Kestelyn, O. Thomas, H. Amano and A. R. Messina, "An Accurate Third-Order Normal Form Approximation for Power System Nonlinear Analysis," IEEE Trans. Power Syst., vol. 33, no. 2, pp. 2128-2139, Mar. 2018.

[19] H. Amano, T. Kumano and T. Inoue, "Nonlinear stability indexes of power swing oscillation using normal form analysis," IEEE Trans. Power Syst., vol. 21, no. 2, pp. 825-834, May 2006.

[20] H. M. Shanechi, N. Pariz and E. Vaahedi, "General nonlinear modal representation of large scale power systems," IEEE Trans. Power Syst., vol. 18, no. 3, pp. 1103-1109, Aug. 2003.

[21] N. Pariz, H. M. Shanechi and E. Vaahedi, "Explaining and validating stressed power systems behavior using modal series," IEEE Trans. Power Syst., vol. 18, no. 2, pp. 778-785, May 2003.

[22] V. I. Arnold, Geometrical Methods in the Theory of Ordinary Differential Equations. New York: Springer-Verlag, 1983.

[23] A. H. Nayfeh, Method of Normal Forms. New York: Wiley, 1993.

[24] R. Genesio, M. Tartaglia, and A. Vicino, "On the estimation of asymptotic stability regions: State of the art and new proposals, " IEEE Trans. Automat. Contr., vol. 30, no. 8, pp. 747-755, Aug. 1985.

[25] R. Genesio and A. Vicino, "Some results on the asymptotic stability of second-order nonlinear systems," IEEE Trans. Automat. Contr., vol. 29, no. 9, pp. 857-861, Sept. 1984.

[26] D. Koditschek and K. Narendra, "The stability of second-order quadratic differential equations," IEEE Trans. Automat. Contr., vol. 27, no. 4, pp. 783-798, Aug. 1982.

[27] M. G. Villalva, J. R. Gazoli and E. R. Filho, "Comprehensive Approach to Modeling and Simulation of Photovoltaic Arrays," IEEE Trans. Power Electron., vol. 24, no. 5, pp. 1198-1208, May 2009.

[28] Y. Xia, M. Yu, P. Yang, Y. Peng and W. Wei, "Generation-Storage Coordination for Islanded DC Microgrids Dominated by PV Generators," IEEE Trans. Energy Convers., vol. 34, no. 1, pp. 130-138, Mar. 2019.

[29] Y. Xia, Y. Peng, P. Yang, M. Yu and W. Wei, "Distributed Coordination Control for Multiple Bidirectional Power Converters in a Hybrid AC/DC Microgrid," IEEE Trans. Power Electron., vol. 32, no. 6, pp. 4949-4959, June 2017. 


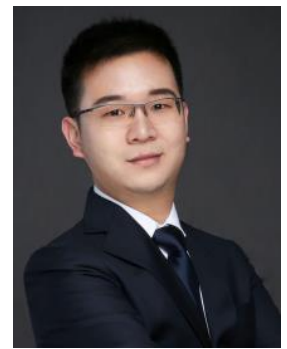

Yanghong Xia (S'16-M'19) received the B.S. degree in automation from the College of Automation, Huazhong University of Science and Technology (HUST), Wuhan, China, and Ph.D degree in control theory and control engineering from the College of Electrical Engineering, Zhejiang University (ZJU), Hangzhou, China, in 2014 and 2019, respectively. Now, he is a Post-doctoral Research Fellow in the joint postdoctoral fellowship program between ZJU and University of Cambridge, Cambridge, U.K.

His current research interests include advanced control methods, stability analysis of power system, hybrid ac/dc microgrids.

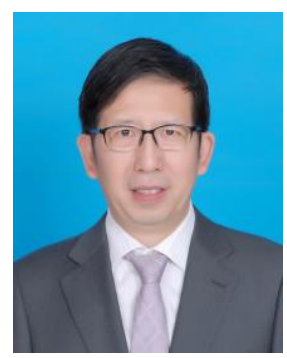

Wei Wei received the B.Eng. degree in automation, M.Eng. degree in control theory and control engineering, and D.Eng. degree in power electronics and electronic drives all from the College of Electrical Engineering, Zhejiang University (ZJU), Hangzhou, China, in 1983, 1986 and 1994, respectively. Now, he is a professor in the College of Electrical Engineering, Zhejiang University.

His current research interests include intelligent control, the development of novel technology of renewable energy and smart grid.

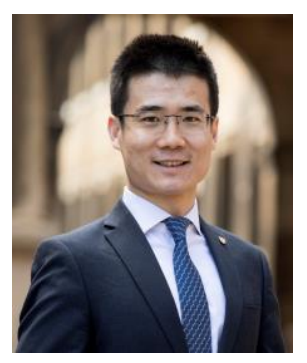

Teng Long (M'13) received the B.Eng. degree from the College of Electrical Engineering, Huazhong University of Science and Technology (HUST), Wuhan, China, the first class B.Eng. (Hons.) degree from the University of Birmingham, Birmingham, U.K., in 2009, and the Ph.D. degree from the University of Cambridge, Cambridge, U.K. in 2013. Until 2016, he was a Power Electronics Engineer with the General Electric (GE) Power Conversion business in Rugby, U.K. He is currently a Lecture with the University of Cambridge.

His current research interests include power electronics, electrical machines, and the machine drives. Dr. Long is a Chartered Engineer (CEng) registered with the Engineering Council in the U.K.

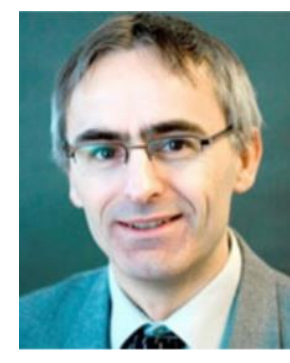

Frede Blaabjerg (S'86-M'88-SM'97F'03) received the Ph.D. degree in electrical engineering from Aalborg University, Aalborg, Denmark, in 1992.

From 1987 to 1988, he was with ABBScandia, Randers, Denmark. In 1992, he became an Assistant Professor, in 1996 an Associate Professor, and in 1998 a Full Professor of power electronics and drives. In 2017, he became a Villum Investigator. He has published more than 450 journal papers in the field of power electronics and its applications. He is the Coauthor of two monographs and the Editor of six books in power electronics and its applications. His research interests include power electronics and its applications such as wind turbines, PV systems, reliability, harmonics, and adjustable speed drives.

Dr. Blaabjerg has received 18 IEEE Prize Paper Awards, the IEEE PELS Distinguished Service Award in 2009, the EPE-PEMC Council Award in 2010, the IEEE William E. Newell Power Electronics Award 2014 and the Villum Kann Rasmussen Research Award 2014. From 2006 to 2012, he was the Editor-in-Chief of the IEEE TRANSACTIONS ON POWER ELECTRONICS. He has been the Distinguished Lecturer of the IEEE Power Electronics Society from 2005 to 2007 and of the IEEE Industry Applications Society from 2010 to 2011 and from 2017 to present. He was nominated in 2014, 2015, and 2016 by Thomson Reuters to be between the most 250 cited researchers in Engineering in the world. In 2017, he became Honoris Causa at University Politehnica Timisoara, Timis, oara, Romania.

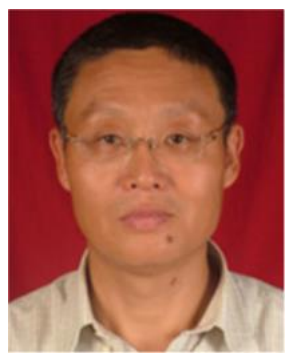

Peng Wang (M'00-SM'11-F'18) received the B.Sc. degree in electrical engineering from Xian Jiaotong University, Xi'an, China, in 1978, the M.Sc. degree in electrical engineering from Taiyuan University of Technology, Taiyuan, China, in 1987, and the M.Sc. and Ph.D. degrees in electrical engineering from the University of Saskatchewan, Saskatoon, SK, Canada, in 1995 and 1998, respectively.

Currently, he is a Professor with the Nanyang Technological University, Singapore. 